\title{
Suppressing Traffic Flow Instabilities
}

\author{
Berthold K.P. Horn
}

\begin{abstract}
While many models of traffic flow predict the instabilities commonly observed-particularly at higher traffic densities-there are few suggestions for suppressing them. A method is described here for suppressing instabilities, thereby reducing gas consumption, accidents, wear and tear on vehicles and roadways as well as travel times while increasing traffic throughput. The method uses information about the following vehicle as well as the leading vehicle. Using information from both sources allows the gain of feedback to be reduced below one, thus eliminating the instability characteristic of "car following." The needed inputs to the control system can be provided by machine vision (or radar or lidar). Previous proposals for smoothing traffic flow instabilities do not use information about the vehicles behind"car following" cruise control methods, for example, focus only on the vehicle ahead. The method presented here is based on information flowing both downstream and upstream, in distinction to traditional approaches where information flows only upstream.
\end{abstract}

\section{BACKGROUND}

We are all only too familiar with traffic flow instabilities, including alternating stop-and-go driving conditions (Fig. 1). These not quite periodic and often rapid variations in speed and traffic density lead to increased danger of collisions, higher fuel consumption, wear and tear on vehicles, faster abrasion of roadways, waste of the drivers' time, and reduction of overall traffic throughput.



Fig. 1. Sample record of traffic flow fluctuations - speed versus time (Interstate 93 North from Boston, 2011 March 31 - from data set collected using roof mounted GPS antenna).

A variety of models of traffic flow predict waves of density and speed moving along roadways [1]-[30], particularly at higher traffic density. Viewed from any particular point on the road, these are large fluctuations in vehicle speed, along with large fluctuations in vehicle density. Viewed from overhead, these waves of density and speed move "upstream" that is, in a direction opposite to that of the traffic flow itself. From the vantage point of a particular vehicle, near periodic variations in vehicle velocity and density are observed as well, although with a different time period than what would be reported by a stationary observer. While there are several ways of explaining the origin of this highly undesirable phenomenon, there have been few suggestions for suppressing it. What is needed is a method for smoothing traffic flow, suppressing travelling waves in density and speed, thereby increasing average speed and overall traffic throughput.

\section{Simple Models that EXPlain The PROBlem}

There are several different ways of explaining how the undesired behavior arises [1]-[30]. These usually start with an assumed relationship between density and speed. Drivers generally attempt to keep a safe distance from the vehicle ahead, that is, a separation large enough so that if the leading car were to suddenly apply its brakes, the following car could apply its brakes in time to avoid a collision, taking into account the finite response time of driver and vehicle. If we assume that the following vehicle applies its brakes after a certain response time, and then decelerates at the same rate as the leading vehicle, then the minimum safe separation can be seen to equal the product of the response time $T$ and the speed $v^{1}$ :

$$
d_{\min }=T v
$$

So, in a situation where vehicles are generally moving at about the same speed, the density (number of vehicles per unit distance) decreases at higher speeds because vehicle spacing increases. If everyone is maintaining a safe separation from the vehicle ahead, then density, $\rho$, is the inverse of the sum of vehicle length $\ell$ and safe separation distance $d_{\min }$ :

$$
\rho=\frac{1}{\ell+T v}
$$

Throughput, $r$, (vehicles passing by a fixed point per unit time) equals density times speed so

$$
r=\frac{v}{\ell+T v}
$$

From this we see that at very low speeds $(v<\ell / T)$, throughput is proportional to speed. Throughput increases with speed but does so more and more slowly as

\footnotetext{
${ }^{1}$ A more detailed analysis takes into account possible differences in the speeds of the vehicles as well.
} 
speed increases. Finally, it would appear that throughput should asymptotically approach

$$
r_{\max }=1 / T
$$

If we took the total response time to be, conservatively, 1 second, for example, then the limiting throughput would be 1 vehicle per second (or 3600 vehicles per hour per lane). This is higher than what is observed in practice.

Throughput is low at low speeds, so high throughput requires higher speeds (consistent with speed limits and considerations of safety). Unfortunately the pleasant scenario of steady increases in throughput with speed is marred by the appearance of instabilities - waves in density and speed that grow in amplitude - often until the speed at the low point of the cycle drops to zero.

One intuitive way of understanding the origin of these instabilities is positive feedback, where lower speed causes higher densities, and higher densities cause even lower speeds, while at the other extreme, higher speed causes lower densities, and lower densities invite even higher speeds. That is, rather than damping out variations from a reasonable average, the system - by its very natureamplifies them. Above some density, a driver applying the brakes can cause the following driver to apply brakes harder and so on, back to a distant point, sometime later, where the traffic may come to a complete stand still (which may occur long after the initial cause has disappeared). A wave travels backward, with amplitude increasing with distance from the original disturbance.

\section{Cascaded control systems}

Another way of understanding where the instabilities come from is to consider each driver and car combination as a control system that adjusts the car's acceleration in response to the relative position of the car ahead, as well as the relative speed of that car. Each driver and car can be thought of as a system with an input (relative position and velocity of the leading vehicle) and an output (acceleration, and hence, indirectly, velocity and position of the vehicle). If there exists any waveform that is amplified by the control system, even if only by a small amount, then cascading many such feedback control systems leads to increasing amplitudes of deviation.

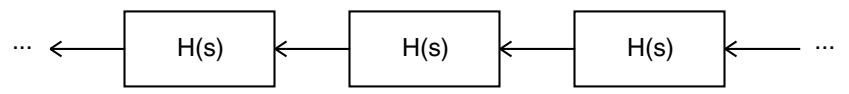

Fig. 2. Cascade of control systems (car following model). Gains greater than one lead to instabilities.

Shown in Fig. 2 is a cascade of control systems ${ }^{2}$ each with transfer function $H(s)$. The overall transfer function

\footnotetext{
${ }^{2}$ The linear model $H(s)$ may be an approximation of a nonlinear system, obtained by expansion about the current operating point. The stability of the system is governed by the behavior of the linearized version. For asymototic stability the poles of $H(s)$ should be in the left half plane.
}

of $n$ such systems is $H^{n}(s)$. If there is some frequency for which the amplitude of the oscillations at the output of a system component is larger than it is at its input (i.e. $|H(j \omega)|>1$ for some $\omega)$, then there will be a problem when many such components are cascaded. That is, if the components have gain greater than one for waves of some frequency, then these gains multiplied together will lead to larger and larger deviations as more and more components are cascaded. So, for stability, the gains need to be strictly less than one for all frequencies. This is the condition for "string stability" [31]-[36].

String stability can be assured through use of "weak coupling" [35] between the control systems. But string stability is not sufficient to guarantee collision-free smooth traffic flow. First consider an extreme example of a system satisfying the string stability condition, namely $H(s)=0$. We can immediately see the problem with this type of control if we consider two vehicles travelling at the same velocity until such time as the leading vehicle slows down. With $H(s)=0$, the trailing vehicle does not respond, and so a collision is unavoidable. The problem is that conditions on the system function $H(s)$ themselves do not provide a means of enforcing positive intervehicle spacing. Naturally, this is a straw man example - no one would actually suggest using a system with $H(s)=0$ - but the problem is more general, as we show next.



Fig. 3. In the simple car-following model, gain less than one leads to path intersection with perturbation.

The upper curve in Fig. 3 shows the position of the leading vehicle as a function of time - relative to the position it would have if it travelled at constant velocity. At a certain point, an oscillatory perturbation of its velocity is imposed, which increases in amplitude with time. The lower curve shows the response of the following vehicle - again relative to the position it would have if it continued to travel at the same constant velocity - in the case when the control system gain is less than one. It is clear that the paths of the two vehicles will intersect for large enough amplitude of perturbation. The illustration can be extended by adding delay to the response, (and adding more vehicles, also with gains less than one), which only serves to make the problem worse. 
Thus the gain cannot be less than one if collisions are to be avoided. Taken together then, the two arguments show that the traffic flow instability problem cannot be solved with the "car following" model of control.

It is also well known that delay in a feedback loop can lead to instabilities [29], [37]. Thus the finite response time of the driver (and the dynamics of the car and its control system) plays a role in the instabilities. Shorter response times allow higher throughput, but for any given response time there will be a critical density above which instabilities are amplified and will propagate.

Finally, system components are non-linear (e.g. speed cannot become negative, cannot exceed some upper limit and the distance between vehicle cannot become negative). Such non-linearities, along with positive feedback, are the classic conditions for chaotic behavior, where small changes in initial conditions can lead to vastly different outcomes and make long range predictions difficult.

\section{Root Cause}

Overall, the root cause of the problem is drivers' feedback control of vehicles. While each driver does what seems reasonable and appears to be best for the progress of their vehicle, the overall effect is clearly not desirable.

Many different models have been made of traffic flow using mathematical tools such as differential and difference equations, cellular automata, fluid flow models, particle tracking, and "car following" models [1]-[30].



Fig. 4. Two vehicles and their relative positions and velocities.

In Fig. 4, traffic flows from left to right, with controlled vehicle ' $\mathrm{C}$ ' and leading vehicle ' $\mathrm{L}$ ' ahead of it. The gap between them is $d_{l}$. The leading vehicle is traveling at speed $v_{l}$ while the controlled vehicle travels at speed $v_{c}$.

Fig. 5 shows the outline of a traditional "car following" control system which produces the acceleration command $a$ for vehicle 'C.' The system takes as input the distance to the leading vehicle, $d_{l}$, and the relative speed of the leading vehicle $\left(v_{l}-v_{c}\right)$. Such a system may also have additional inputs, such as the speed of the vehicle $v_{c}$ itself, as well as parameters that control the operation, such as the desired speed $v_{\text {des }}$ and the speed limit $v_{\max }$. The output of the control system is the acceleration command $a$ sent to the drive control system. Alternatively it could be a speed setpoint for the drive control system. Negative acceleration commands may activate mechanical brakes and/or a regenerative braking system [38], [39].



Fig. 5. A simple car following control system.



Fig. 6. Illustrative car following control system.

A more detailed diagram of an illustrative car following control system is shown in Fig. 6. Here $k_{d}$ is the gain for feedback based on the difference between the distance to the leading car and the desired distance $d_{\mathrm{des}}$ (which may vary with $v_{c}$ as indicated above). Further, $k_{v}$ is the gain for the component of feedback based on the relative velocity $\left(v_{l}-v_{c}\right)$. In addition, $k_{c}$ is the gain for the component of feedback based on the difference between the desired velocity $v_{\text {des }}$ and the current velocity $v_{c}$. Finally, a separate circuit vetoes positive acceleration when the current velocity $v_{c}$ is at the speed limit $v_{\max }$.

In a simple, linearized, version of this system, acceleration may be given by

$$
a=k_{d}\left(d_{l}-d_{\mathrm{des}}\right)+k_{v}\left(v_{l}-v_{c}\right)+k_{c}\left(v_{c}-v_{\mathrm{des}}\right)
$$

This model and others like it suffer from travelling waves and amplification of perturbations above some critical density. None of these models directly suggest a solution to the problem of traffic flow instabilities.

An important thing to note is that information flow in such a chained feedback system is strictly one-way: from front to back. Each vehicle's acceleration is adjusted based on information of the relative position and velocity of the 
vehicle in front of it. Each vehicle only influences what is behind it. There is no information propagating forward in traffic. The result is reminiscent of the fluid dynamic phenomena of a shock wave, where no information moves forward across the shock [4].

\section{Prior Suggestions for Improving Traffic Flow}

Since the incidence of traffic flow instabilities increases with traffic flow density, one approach to the problem of limiting traffic flow instabilities is to keep density low. More roads and more lanes per road certainly help reduce the density, but are in many cases no longer a viable alternative from land use and economic perspectives.

Limiting access, or "metering," at freeway entrances so as to keep the density on the freeway at or below some target value certainly helps [40], but forces the freeway to operate below its maximum carrying capacity.

Another approach is to reduce the response time, thus allowing vehicles to follow each other more closely at high speed without danger of collision. The standard arrangement of accelerator and brake pedal makes the response time unnecessarily long since the foot has to be lifted off one and applied to the other, but it is unlikely that much can be done about this misfeature now [41].

Instead, an automated control system somewhat analogous to cruise control, but using automatic feedback based on sensor readings, can reduce the effective "response time." But as pointed out above, there is still a critical density above which instabilities occur. There is also some question about what happens when vehicles with such automated control are mixed with vehicles directly controlled by drivers. To the human drivers, these automated vehicles will appear to be "tail gating" at uncomfortably close range.

Fully automated control systems with high speed communication between vehicles can allow a lead vehicle to directly control several following vehicles, allowing them to travel together in "platoons" much as the engine of a train and the carriages tied to it. However, such an approach is best suited for a separate road system limited to platoons of fully automated vehicles. It is unlikely to be safe in a "mixed" environment with some vehicles controlled by human drivers.

Suggestions for automatic "distance keeping" from the leading vehicle go back at least to the work of Dr. Ichiro Masaki [38], then at G.M. Technical Center (see e.g. U.S. patent 4,987,357). His advanced cruise-control system, which automatically adjusts the car's speed to keep a safe separation from vehicles in front, is based on machinevision technology. The system detects the presence of a car directly in front using a video camera, and stores a reference image. The stream of incoming camera images are then compared with the stored image as the cars move down the highway. Because the image of the car being followed will become larger or smaller-depending on how close the car is - the system can adjust the distance between the cars so as to match the scale of the stored image. Dr. Masaki also suggested using machine vision methods based on binocular stereo from pairs of cameras as an alternative method for estimating the distance.

Such suggestions for "adaptive cruise control" rely on information about the leading vehicle, typically the distance to that vehicle, and perhaps the relative speed.

\section{Bilateral Control}

One way to underestand the "trick" to solving the problem is to consider a way of obtaining enough overall gain in each control system to prevent collisions, yet avoid a gain greater than one in any individual pathway from an input to an output. One way to achieve this is for the control system to take into account not only the car ahead, but the car behind. That is, allow information to flow in both directions, not just from front to back ${ }^{3}$.



Fig. 7. Three vehicles and their positions and velocities.

More generally, in bilateral control (see Fig. 7), the acceleration of the controlled vehicle ' $\mathrm{C}$ ' is dependent on the distance $d_{l}$ to the leading vehicle ' $\mathrm{L}$ ', the relative speed $\left(v_{l}-v_{c}\right)$ of the leading vehicle, as well as the distance $d_{f}$ of the following vehicle and the relative speed $\left(v_{c}-v_{f}\right)$ (compare this to the car following model shown in Fig. 4).

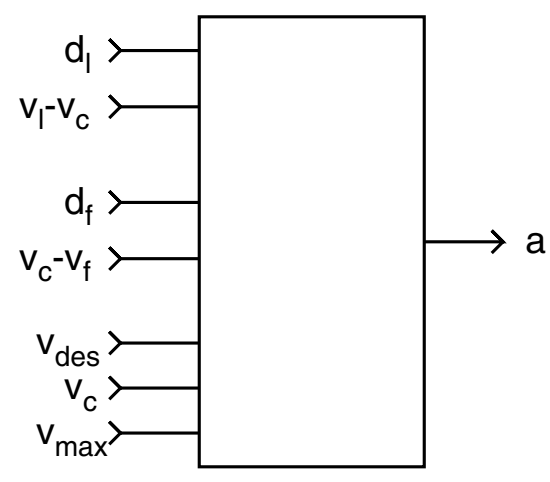

Fig. 8. A system for bilateral control.

Fig. 8 illustrates the bilateral control system which takes as additional inputs the distance from the following vehicle, $d_{f}$, and the relative velocity of the following vehicle $\left(v_{f}-v_{c}\right)$. Auxiliary inputs may include signals that indicate whether there is in fact a vehicle ahead and whether there is a vehicle following.

\footnotetext{
${ }^{3} \mathrm{~A}$ simplistic version of this idea is to have a vehicle attempt to maintain a position halfway between that of the vehicle ahead and that of the vehicle behind.
} 
Here the acceleration $a$ is a function of the distance to the leading vehicle $d_{l}$, the relative velocity $\left(v_{l}-v_{c}\right)$, and the distance $d_{f}$ to the following vehicle, as well as the relative velocity $\left(v_{f}-v_{c}\right)$. As before, acceleration may also depend on the desired velocity $v_{\text {des }}$, the current velocity $v_{c}$ of the car being controlled, and a speed limit $v_{\max }$ (compare this to the car following model shown in Fig. 5).

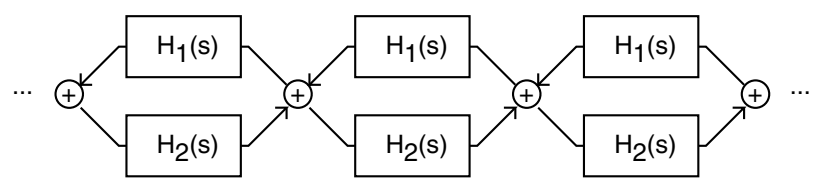

Fig. 9. Cascade of control systems (bilateral control).

Shown in Fig. 9 is a cascade of control systems, each with backward transfer function $H_{1}(s)$ and forward transfer function $\mathrm{H}_{2}(s)$ (compare to Fig. 2 for the car following model). If the gain of the control system is around 1/2 when measured from the leading car input and also around $1 / 2$ when measured from the following car input, then there is enough overall gain to avoid collisions, while the gain going from front to back or from back to front is less than one (namely about 1/2), thus attenuating any oscillations or travelling waves. Perturbations are dissipated by being forced both forward and backward.

\section{Physical model of Bilateral CONTROL}

A simple physical model will make this counterintuitive result appear more plausible. Adjustment of acceleration based on distance can be modeled by a spring connecting cars, since, by Hooke's law, the force in a spring is proportional to the extension from its rest length, and by Newton's second law of motion, the acceleration is the force divided by the mass of the car. We also need a force proportional to the relative velocity of the cars, which can be produced by a damper or shock absorber.



Fig. 10. Mechanical analog model of bilateral control systems.

Consider such a mechanical mass/spring/damper analog of a control system with bilateral feedback (Fig. 10). Here the masses (modeling the vehicles) are connected to each other with springs that transmit a force proportional to the difference between the spacing of the vehicles and the resting length of the spring. In addition, damping is provided by dashpots or shock absorbers that produce a force proportional to the speed of compression or expansion, in the direction opposite to that of the motion.

Suppose now that one grabs hold of one mass in such a string of masses, springs and dampers. If one moves that mass periodically in the direction of the length of the chain to simulate a perturbation, that motion will be transmitted along the chain, but will be damped out along the chain by the mass/spring/damper combination. This damping may work better at some frequencies than at others, but there will never be an increase in amplitude along the chain for any frequency. For one thing, in such a purely passive system (i.e. one without active components that could amplify signals) there are no energy sources to support increasing amplitudes of motion.

In fact, the differential equation describing the system can be shown to be the one-dimensional damped wave equation - provided a suitable choice of coordinates is made. It can also be shown that such a system will approach a steady state, with vehicle speeds converging to a common value, and vehicle separation approaching a common value (see Appendix I).

One might wonder why this physical model does not apply to the simple "car following" control systems described earlier. In that system, it would appear that a spring and a damper could model a control system where acceleration is proportional to distance and relative velocity with respect to the car ahead. But note that by Newton's third law, every action has an equal and opposite reaction, which here means that the springs and dampers exert forces not just on the following car, as appropriate for "car following," but also on the one ahead. But there is nothing comparable to this in the "car following" model, since in it, the driver ahead pays no heed to the car behind.

An interesting way of looking at the method is to note how it "cools" the relative motions in a group of vehicles. Suppose that a number of vehicles using this new type of control system start off with different initial velocities and different inter-vehicle spacing. The effect of the "damper" part of the control system is to dissipate the kinetic and potential energy resulting from departures from the average, thus reducing the difference between individual vehicle's motions and the average.

One can compare such a group of vehicles to molecules in a gas flow. Individual molecules move relative to the average in a way that depends on the temperature of the gas - the hotter the gas the more rapid the relative motion. Dropping the temperature corresponds to reducing the differences between the velocities of individual molecules and the average velocity of the group of molecules. As the gas is cooled it may undergo a phase change, such as changing from gaseous to liquid form (see also [14], [15], [42]). Similarly, a group of vehicles using this type of control may enter a phase in which the vehicles are all travelling at essentially the same speed and the intervehicle spacings are essentially the same.

Note that such a "cool" phase of a group of vehicles is different from that of a "platoon" of cars in that: (i) there is no direct communication between the lead car and the other cars, (ii) no single driver is in control of the group, (iii) it is easy for cars to drop out of the group and other cars to be added, (iv) the group of cars can 
easily react to disturbances, and, afterwards, once more drift back towards a "cool" equilibrium phase.

\section{IMPLEMENTATION}

There remains an issue of how to implement the control system. First, drivers could just start paying attention to the car following them and not follow the car ahead as closely if there is a gap behind them. A particular simple version of this notion would have each driver aim to be half way between the car behind and the car ahead (think of one spring connecting the car to the one ahead and another to the one behind). The driver would also need to take into account the speed relative to the average speed of the car behind and the car ahead (and slow down if the speed was higher, or accelerate if it was lower).

This simple solution would damp out traffic flow instabilities, but would require a large fraction of drivers to adopt an unfamiliar driving mode. Further, paying attention to the car behind takes attention away from the car ahead and thus most likely reduces safety ${ }^{4}$.

Some form of automation is preferable, where sensors determine the relative positions and speeds of the vehicles ahead and behind, and use this information to automatically control the acceleration of the vehicle.

In one simple form, the system can operate much in the way of a cruise control system, except, instead of maintaining a fixed speed, the system follows the bilateral control law. Very roughly speaking, the appearance will be that of a vehicle attempting to match the average speed of the leading and the following vehicle while staying more or less in the middle between them (the precise details of operation are as defined above for bilateral control).

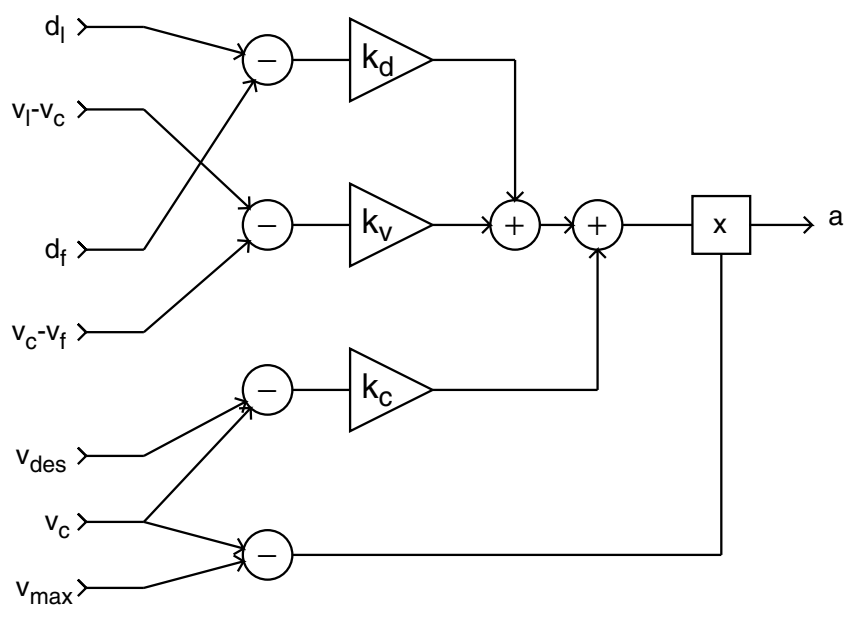

Fig. 11. Illustrative bilateral control system.

A diagram of a sample bilateral control system is shown in Fig. 11. Here $k_{d}$ is the gain for feedback based on the difference of the distances to the leading and the

\footnotetext{
${ }^{4}$ Also, some drivers are likely to be loath to leave gaps ahead that non-cooperating drivers from other lanes may populate.
}

following cars, while $k_{v}$ is the gain for feedback based on the difference between the relative velocities $\left(v_{l}-v_{c}\right)$ and $\left(v_{c}-v_{f}\right)$. In addition, $k_{c}$ is the gain of feedback based on the difference between the desired velocity $v_{\text {des }}$ and the current velocity $v_{c}$, while a separate circuit vetoes positive acceleration when the current velocity $v_{c}$ is equal to the speed limit $v_{\max }$ (compare with Fig. 6. for car following model). In a simple linearized version of this system, acceleration may be given by

$$
a=k_{d}\left(d_{l}-d_{f}\right)+k_{v}\left(\left(v_{l}-v_{c}\right)-\left(v_{c}-v_{f}\right)\right)+k_{c}\left(v_{c}-v_{\text {des }}\right)
$$

There would also be additional control rules that take into account minimum safe separation, relative speeds, speed limits, weather and lighting conditions, traffic density and traffic advisories. Special rules could deal with the situation when there is in fact no vehicle ahead and/or no vehicle behind. The control system can, for example, have multiple states, s.a. (1) both leading and following vehicles present, (2) no following vehicle, (3) no leading vehicle, and (4) no following or leading vehicle. If there are no nearby vehicles, it could revert to a simple cruise control mode where the acceleration is simply a function of the current speed and the desired speed.

The feedback shown is linear, but non-linear feedback is also a possibility, including "gain scheduling" or "switching mode" feedback. In all of these versions the common key idea here is that information about distance and speed of the vehicle following is also taken into account.

The sensors needed to implement this type of control system would be in the vehicle ${ }^{5}$. The vehicle could, for example, have cameras aimed fore and aft able to estimate distance and velocity to the nearest vehicle, using e.g. machine vision methods such as "optical flow," "time to contact," and perhaps binocular or trinocular stereo [38], [39], [43]-[47]. Laser, ultrasound and radar measurement technique could be used instead, although likely more expensive and less able to discriminate between vehicles in the same lane and those in adjacent lanes.

A system augmenting bilateral control with communication between adjacent vehicles has been described recently [48]. This makes possible strong proofs of stability even in the presence of failures of system components.

\section{Simulation}

The "car following" model and the "bilateral control" model have been implemented in simulation and tested under various conditions and with various gain and parameter settings. These simulations confirm the appearance of traffic flow instabilities under a wide variety of conditions when using the car following model. The feedback systems for "car following" and "bilateral control" were based on what is presented here (Fig. 6 and 11), with some minor additions, such as a more sophisticated method for calculating the "minimum safe separation" for

\footnotetext{
${ }^{5}$ It would be much less desirable to depend on sensors in supporting infrastructure.
} 




Fig. 12. Unstable traffic flow using car following model.

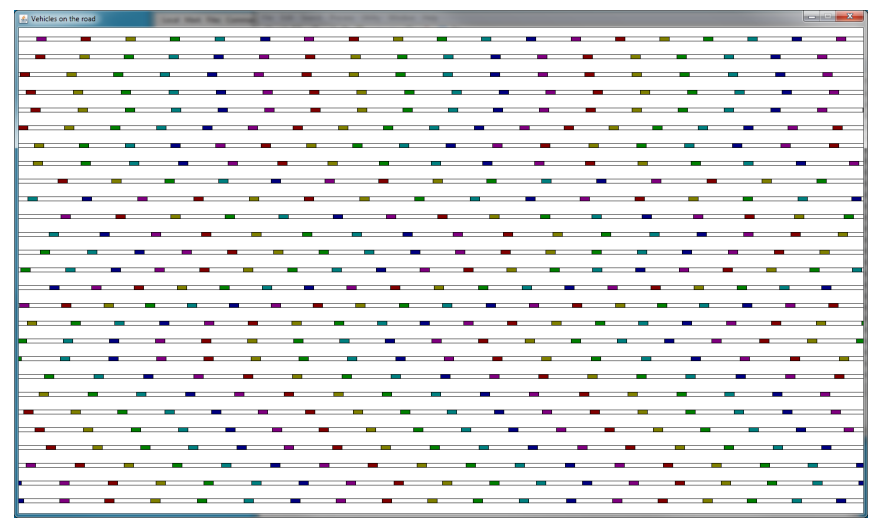

Fig. 13. Stable traffic flow using bilateral control.

the car following model that takes into account differences in speed as well as position, and a system for braking hard when the gap between vehicles should ever become less than the minimum safe separation.

A java application simulating both the "car following" and the "bilateral control" system can be downloaded from http://csail.mit.edu/ bkph/Traffic_Flow_ Instabilities This application provides for experimentation with variation in parameters. Sample screen shots show results after running the "car following" system for a few minutes (Fig. 12), and then switching to bilateral control for a few minutes (Fig. 13) ${ }^{6}$.

\section{WHY THIS SHOULD BE IMPLEMENTED}

If most cars had this kind of system, then traffic would flow more smoothly at high densities, and existing roadways could sustain higher throughputs. Gas consumption would be reduced, as will time lost sitting in stop-and-go traffic. Wear and tear on roads and vehicles would be lessened and the incidence of accidents reduced. Community benefits could be significant, since more traffic could be accommodated without adding to the infrastructure.

\footnotetext{
${ }^{6}$ For the default settings of this particular implementation, the car-following mode leads to an average speed of $15.4 \mathrm{~m} / \mathrm{sec}$ (st. dev. $7.8 \mathrm{~m} / \mathrm{sec}$ ) and a throughput of 0.77 cars $/$ second, while with bilateral control the average speed rises to $25 \mathrm{~m} / \mathrm{sec}$ (st. dev. $0.15 \mathrm{~m} / \mathrm{sec}$ ) and the throughput to $1.25 \mathrm{car} / \mathrm{sec}$.
}

\section{REFERENCES}

[1] B. D. Greenshields, "A study of traffic capacity," Proc. of the Highway Research Board, vol. 14, pp. 448-477, 1935.

[2] M. Lighthill and G. B. Whitham, "On kinematic waves: A theory of traffic flow on long crowded roads," Proc. of the Royal Society - series A, vol. 229, no. 1178, pp. 317-345, May 1955.

[3] G. F. Newell, "Mathematical models for freely-flowing highway traffic," Operations Research, vol. 3, no. 2, pp. 176186, May 1955.

[4] P. I. Richards, "Shock waves on the highway," Operations Research, vol. 4, pp. 42-51, 1956.

[5] L. C. Edie and R. S. Foote, "Traffic flow in tunnels," Proc. of the Highway Research Board, vol. 37, pp. 334-344, 1958.

[6] H. Greenberg, "An analysis of traffic flow," Operations Research, vol. 7, no. 1, Jan/Feb 1958.

[7] R. E. Chandler, R. Herman, and E. W. Montroll, "Traffic dynamics: Studies in car following," Operations Research, vol. 6 , no. 2 , pp. $165-184,1958$.

[8] R. Herman, E. W. Montroll, R. B. Potts, and R. W. Rothery, "Traffic dynamics: Analysis of stability in car following," Operations Research, vol. 7, no. 1, pp. 86-106, 1959.

[9] L. C. Edie, "Car-following and steady-state theory for noncongested traffic," Operations Research, vol. 9, no. 1, pp. 66-75, 1961.

[10] I. Prigogine and R. Herman, Kinetic Theory of Vehicular Traffic. New York: Elsevier, 1971.

[11] J. Treiterer and J. A. Myers, "Hysteresis phenomenon in traffic flow," in Proc. of the 6th Int. Symp. on Transportation and Traffic Theory, D. Buckley, Ed. New York: Elsevier, 1974, pp. 13-38.

[12] K. Nagel and M. Schreckenberg, "A cellular automaton model for freeway traffic," Journal of Physics I (France), vol. 2 , p. $2221,1992$.

[13] G. F. Newell, "A simplified theory of kinematic waves in highway traffic, I. general theory, II. queuing at freeway bottlenecks, III. multidestination flows," Transportation Research, vol. 27B, pp. 281-313, 1993.

[14] B. S. Kerner and H. Rehborn, "Experimental properties of phase transitions in traffic flow," Physical Review Letters, vol. 79 , p. 4030, 1997.

[15] C. F. Daganzo, M. Cassidy, and R. L. Bertini, "Possible explanations of phase transitions in highway traffic," Transportation Research Part A, vol. 33, p. 365, 1999.

[16] D. Helbing and M. Schreckenberg, "Cellular automata simulating experimental properties of traffic flow," Physical Review E, vol. 59, p. R2505, March 1999.

[17] K. Nagel, "Hell on wheels," The Sciences, p. 26, 1999. [Online]. Available: http://www.shaav.com/professional/ shad/Sciences9905-_HellonWheels.pdf

[18] P. Weiss, "Stop-and-go science," Science News, vol. 156, no. 1, pp. 8-10, July 1999. [Online]. Available: http://www.sciencenews.org/sn_arc99/7_3_99/bob1.htm

[19] P. Ball, "Slow, slow, quick, quick, slow," Nature Science Update, vol. 20, pp. 1858-1864, April 2000. [Online]. Available: http://www.nature.com/news/2000/ 000420/full/news000420-_4.html

[20] T. Nagatani, "Traffic jams induced by fluctuation of leading car," Physical Review E, vol. 61, pp. 3534-3540, April 2000.

[21] I. Peterson, "Traffic woes of the single driver," Science News, vol. 157, p. 303, May 2000.

[22] E. Tomer, L. Safonov, and S. Havlin, "Presence of many stable nonhomogeneous states in an inertial car-following model," Physical Review Letters, vol. 84, pp. 382-385, Jan 2000.

[23] I. Peterson, "Math trek: Waves of congestion," Science News, vol. 221, Sep 2001. [Online]. Available: http: //www.maa.org/mathland/mathtrek_9_10_01.html

[24] D. Gazis, "The origins of traffic theory," Operations Research, vol. 50, no. 1, pp. 69-77, Jan/Feb 2002.

[25] S. Begley, "A few computer-controlled cars can help traffic," Wall Street Journal, July 2004. 
[26] L. C. Davis, "Effect of adaptive cruise control systems on traffic flow," Physical Review E, vol. 69, June 2004.

[27] J. R. Minkel, "The computer minds the commuter," Physical Review Focus, June 2004. [Online]. Available: http://focus.aps.org/story/v13/st26

[28] I. Peterson, "Math trek: Cruise control and traffic flow," Science News, Nov 2004. [Online]. Available: http://www.maa.org/mathland/mathtrek_11_22_04.html

[29] G. Orosz and G. Stépán, "Subcritical hopf bifurcations in a car-following model with reaction-time delay," Proc. of the Royal Society A, vol. 462, 2006.

[30] J. A. Laval and L. Leclerq, "A mechanism to describe the formation and propagation of stop-and-go waves in congested freeway traffic," Philosophical Trans. Royal Society $A$, vol. 368(1928), pp. 4519-4541, 2010.

[31] S. Sheikholesam and C. A. Desoer, "Longitudinal control of a platoon of vehicles," in Proc. 1990 ACC, San Diego, CA, 1990.

[32] C. C. Chien and P. Ioannou, "Automatic vehiclefollowing," in Proc. of the American Control Conference, Chicago, IL, June 1992, pp. 1748-1752.

[33] P. Ioannou and C. C. Chien, "Autonomous intelligent cruise control," IEEE Trans. on Vehicular Technology, vol. 42, no. 4, pp. 657-672, November 1993.

[34] P. Ioannou and $\mathrm{Z}$. Xu, "Throttle and brake control systems for automatic vehicle following," IVHS Journal, vol. 1, no. 4, 1994.

[35] D. Swaroop and J. K. Hedrick, "String stability of interconnected systems," IEEE Trans. Automatic Control, vol. 41, no. 3, pp. 349-356, March 1996.

[36] C.-Y. Liang and H. Peng, "Optimal adaptive cruise control with guaranteed string stability," Vehicle System Dynamics, vol. 31, pp. 313-330, 1999.

[37] L. C. Davis, "Modification of the optimal velocity traffic model to include delay due to driver reaction time," Physica $A$, vol. 319, 2003.

[38] I. Masaki, "Vision-based mobile robots on highways," Advanced Robotics, vol. 9, no. 4, pp. 417-427, 1995.

[39] J. Leonard and et al., "A perception-driven autonomous vehicle," Journal of Field Robotics, vol. 25, no. 10, pp. 727774 , Oct 2008.

[40] L. Zhang and D. Levinson, "Ramp metering and freeway bottleneck capacity," Transportation Research Part A, pp. 218-235, 2010.

[41] R. E. Fenton and W. B. Montano, "An intervehicular spacing display for improved car-following performance," IEEE Trans. on Man-Machine Systems, vol. MMS-9, June 1968.

[42] M. Treiber, A. Kesting, and D. Helbing, "Three-phase traffic theory and two-phase models with a fundamental diagram in the light of empirical stylized facts," Transportation Research Part B: Methodological, vol. 44, pp. 983$1000,2010$.

[43] M. Bertozzi and A. Broggi, "Gold: a parallel real-time stereo vision system for generic obstacle and lane detection," IEEE Trans. on Image Processing, vol. 7, no. 1, pp. 62-80, June 1998.

[44] J. C. McCall and M. M. Trivedi, "Video-based lane estimation and tracking for driver assistance: Survey, system, and evaluation," IEEE Trans. on Intelligent Transportation Systems, vol. 7, no. 1, pp. 20-37, March 2006.

[45] B. K. P. Horn, Y. Fang, and I. Masaki, "Time to contact relative to a planar surface," IEEE Trans. on Intelligent Transportation Systems, vol. 7, no. 1, June 2007.

[46] A. S. Huang, "Lane estimation for autonomous vehicles using vision and lidar," Ph.D. dissertation, MIT, Feb 2010.

[47] M. Bertozzi, A. Broggi, and A. Fascioli, "Vision-based intelligent vehicles: State of the art and perspectives," Robotics and Autonomous Systems, vol. 1, pp. 1-16, 2000.

[48] T. Baran and B. K. P. Horn, "A robust signal-flow architecture for cooperative vehicle density control," in Proc. of the 38th Int. Conference on Acoustics, Speech, and Signal Processing. Vancouver, Canada: IEEE Signal Processing Society, May 2013.
APPENDIX I

Proof OF STABILITY OF SIMPLE LINEAR BILATERAL CONTROL

Let

$$
E=\frac{1}{2} \sum_{n=0}^{N-1} \dot{x}_{n}^{2}+\frac{1}{2} k_{d} \sum_{n=0}^{N-1}\left(x_{n+1}-x_{n}\right)^{2}
$$

We will show that $E$ decreases with time and that it does so until the velocities of the vehicles match.

In the above expression for $E$, consider $N$ vehicles on a closed loop, where the indexes wrap around, with vehicle number $N$ being the same as vehicle number 0 . We show next that the distance between vehicles approaches a common value equal to the length of the track divided by $N$. We have

$$
\frac{d E}{d t}=\sum_{n=0}^{N-1} \dot{x}_{n} \ddot{x}_{n}+k_{d} \sum_{n=0}^{N-1}\left(x_{n+1}-x_{n}\right)\left(\dot{x}_{n+1}-\dot{x}_{n}\right)
$$

The second sum equals

$$
-k_{d} \sum_{n=0}^{N-1} \dot{x}_{n}\left(x_{n+1}-2 x_{n}+x_{n-1}\right)
$$

if the index $n$ is taken $\bmod N$. Now, in the case of the simple linear bilateral control system,

$$
\ddot{x}_{n}=k_{d}\left(x_{n+1}-2 x_{n}+x_{n-1}\right)+k_{v}\left(\dot{x}_{n+1}-2 \dot{x}_{n}+\dot{x}_{n-1}\right)
$$

So the first sum in the expression for $d E / d t$ becomes

$$
k_{d} \sum_{n=0}^{N-1} \dot{x}_{n}\left(x_{n+1}-2 x_{n}+x_{n-1}\right)+k_{v} \sum_{n=0}^{N-1} \dot{x}_{n}\left(\dot{x}_{n+1}-2 \dot{x}_{n}+\dot{x}_{n-1}\right)
$$

The first term in this expression is cancelled by the second sum in the expression for $d E / d t$, so that $d E / d t$ simplifies to

$$
\frac{d E}{d t}=k_{v} \sum_{n=0}^{N-1} \dot{x}_{n}\left(\dot{x}_{n+1}-2 \dot{x}_{n}+\dot{x}_{n-1}\right)
$$

or

$$
\frac{d E}{d t}=-k_{v} \sum_{n=0}^{N-1}\left(\dot{x}_{n+1}-\dot{x}_{n}\right)^{2}
$$

if the index $n$ is taken $\bmod N$. So $d E / d t \leq 0$ and $d E / d t=0$ only when all vehicles have the same velocity. That is the state that the system converges to. Convergence clearly does require velocity feedback, and is faster when $k_{v}$ is higher.

Further, when all the velocities are the same, the contribution to the commanded acceleration from velocity feedback is zero, leaving only the contribution from positional feedback. So any remaining acceleration must be due to differences in spacing of the vehicles. But if the velocities remain matched, acceleration must be zero, so the differences in spacing between vehicles must approach zero also. Hence, not only do the vehicles asymptotically approach the same common velocity, but the spacing between them also approaches a common value. 